\title{
MS09-01 | Tools To Aid Macromolecular Refinement At Low Resolution
}

Nicholls, Robert (Medical Research Council, Cambridge, GBR); Emsley, Paul (Medical Research Council, Cambridge, GBR); Kovalevskiy, Oleg (Medical Research Council, Cambridge, GBR); Long, Fei (Medical Research Council, Cambridge, GBR); Murshudov, Garib (Medical Research Council, Cambridge, GBR)

Effects such as crystal mosaicity and disorder lead to poor diffraction quality and weak intensities, resulting in only low-resolution data being available. Low observation-to-parameter ratio and suboptimal parameterisation cause unstable refinement, overfitting, and ultimately result in an unreliable model.

Regularisers stabilise macromolecular refinement, ensuring consistency between derived models and prior knowledge. Restraints representing chemical information help local structure adopt chemically reasonable conformations. At lower resolutions, supplementary restraints are used: encouraging consistency with models of homologous structures, formation of hydrogen bonding networks, nucleotide base pairing and stacking. Jelly-body restraints stabilise model refinement without injecting externally derived information. An anharmonic penalty function is used in order to control robustness to outliers due to inconsistencies between data and prior. Additional restraints and robust estimation are also useful for model building, increasing real space refinement convergence radius and stability.

Where several datasets and models are available for a macromolecule, restraints can facilitate information transfer between structures, potentially improving refinement and thus resultant model quality. Pragmatically, determining suitability of reference structures and refinement parameters can be challenging; the automated pipeline LORESTR facilitates this process by trialling multiple protocols.

This contribution will discuss techniques to facilitate high-quality models being routinely achieved in cases where only low-resolution data are available, focussing on implementation in REFMAC5, Coot, ProSMART, LibG and LORESTR. Other developments in refinement, map calculation, analysis and validation tools will also be discussed.

[1] Nicholls R.A., Kovalevskiy O. and Murshudov G.N. (2017) Low resolution refinement of atomic models against crystallographic data. Protein Crystallography: Methods and Protocols, 565-93. 